\title{
DUST-OBSCURED STAR FORMATION IN INTERMEDIATE REDSHIFT GALAXY CLUSTERS
}

\author{
Rose A. Finn ${ }^{1}$, Vandana Desai ${ }^{2,3}$, Gregory Rudnick ${ }^{4,5}$, Bianca Poggianti ${ }^{6}$, Eric F. Bell ${ }^{7,8}$, Joannah Hinz $^{9}$, \\ Pascale Jablonka $^{10}$, Bo Milvang-Jensen ${ }^{11}$, John Moustakas ${ }^{12}$, Kenneth Rines ${ }^{13}$, and Dennis Zaritsky ${ }^{9}$ \\ ${ }^{1}$ Department of Physics, Siena College, 515 Loudon Road, Loudonville, NY 12211, USA; rfinn@ siena.edu \\ ${ }^{2}$ Division of Physics, Mathematics and Astronomy, California Institute of Technology, Pasadena, CA 91125, USA \\ ${ }^{3}$ Spitzer Science Center, California Institute of Technology, Pasadena, CA 91125, USA \\ ${ }^{4}$ Department of Physics and Astronomy, University of Kansas, Malott Hall, Room 1082, 1251 Wescoe Hall Drive, Lawrence, KS 66045, USA \\ ${ }^{5}$ National Optical Astronomy Observatory, Tucson, AZ 85719, USA \\ ${ }^{6}$ Osservatorio Astronomico, vicolo dell'Osservatorio 5, 35122 Padova, Italy \\ ${ }^{7}$ Max-Planck-Institut für Astronomie, Königstuhl 17, D69117 Heidelberg, Germany \\ ${ }^{8}$ Department of Astronomy, University of Michigan, 500 Church Street, Ann Arbor, MI 48105, USA \\ ${ }^{9}$ Steward Observatory, 933 North Cherry Avenue, University of Arizona, Tucson, AZ 85721, USA \\ 10 Observatoire de lUniversité de Genève, Laboratoire dAstrophysique de lEcole Polytechnique Fédérale de Lausanne (EPFL), 1290 Sauverny, Switzerland \\ ${ }^{11}$ Dark Cosmology Centre, Niels Bohr Institute, University of Copenhagen, Juliane Maries Vej 30, DK-2100 Copenhagen Ø, Denmark \\ ${ }^{12}$ Center for Astrophysics and Space Sciences, University of California, San Diego, 9500 Gilman Drive, La Jolla, CA 92093, USA; jmoustakas@ ucsd.edu \\ ${ }^{13}$ Department of Physics and Astronomy, Western Washington University, Bellingham, WA 98225, USA; kenneth.rines@wwu.edu \\ Received 2009 November 20; accepted 2010 July 5; published 2010 August 5
}

\begin{abstract}
We present Spitzer MIPS $24 \mu \mathrm{m}$ observations of sixteen $0.4<z<0.8$ galaxy clusters drawn from the ESO Distant Cluster Survey. This is the first large $24 \mu \mathrm{m}$ survey of clusters at intermediate redshift. The depth of our imaging corresponds to a total IR luminosity of $8 \times 10^{10} L_{\odot}$, just below the luminosity of luminous infrared galaxies (LIRGs), and $6_{-1}^{+1} \%$ of $M_{V}<-19$ cluster members show $24 \mu \mathrm{m}$ emission at or above this level. We compare with a large sample of coeval field galaxies and find that while the fraction of cluster LIRGs lies significantly below that of the field, the IR luminosities of the field and cluster galaxies are consistent. However, the stellar masses of the EDisCS LIRGs are systematically higher than those of the field LIRGs. A comparison with optical data reveals that $\sim 80 \%$ of cluster LIRGs are blue and the remaining $20 \%$ lie on the red sequence. Of LIRGs with optical spectra, $88_{-5}^{+4} \%$ show $[\mathrm{O} \mathrm{II}]$ emission with $\mathrm{EW}([\mathrm{O} \mathrm{II}])>5 \AA$, and $\sim 75 \%$ exhibit optical signatures of dusty starbursts. On average, the fraction of cluster LIRGs increases with projected clustercentric radius but remains systematically lower than the field fraction over the area probed $\left(<1.5 \times R_{200}\right)$. The amount of obscured star formation declines significantly over the 2.4 Gyr interval spanned by the EDisCS sample, and the rate of decline is the same for the cluster and field populations. Our results are consistent with an exponentially declining LIRG fraction, with the decline in the field delayed by $\sim 1$ Gyr relative to the clusters.
\end{abstract}

Key words: galaxies: clusters: general - galaxies: evolution

\section{INTRODUCTION}

Rest-frame ultraviolet and optical studies indicate that the global star formation rate (SFR) density has decreased by a factor of approximately 10 between $z=0$ and $z=2$ (Gallego et al. 1995; Lilly et al. 1996; Madau et al. 1996; Connolly et al. 1997; Treyer et al. 1998; Flores et al. 1999; Steidel et al. 1999; Wilson et al. 2002; Giavalisco et al. 2004; Hopkins 2004). What drives the decline in SFR? Several scenarios have been proposed, including the depletion of cold gas due to continuous star formation and/or merger-driven bursts (e.g., Bekki \& Couch 2003); a decrease in the rate of galaxy-galaxy interactions that trigger star formation (e.g., Le Fèvre et al. 2000; Bridge et al. 2007; but see also Lotz et al. 2008); and the quenching of star formation in galaxies entering increasingly dense environments as structure forms in the universe (e.g., Larson et al. 1980).

Many of the mechanisms that fall within the above categories result not only in a decline in the global SFR, but also in an environmental dependence on SFR. Indeed, it is well established that the SFRs of galaxies in the local universe correlate with the environment, in the sense that high-density environments have a lower fraction of star-forming galaxies than low-density environments (e.g., Hashimoto et al. 1998; Lewis et al. 2002; Gómez et al. 2003; Balogh et al. 2004; Kauffmann et al. 2004; Blanton \& Moustakas 2009). Large spectroscopic and photometric redshift surveys of the general field have allowed the study of the SFR-density relation out to $z \approx 1$. Such studies have been undertaken in GOODS (Elbaz et al. 2007), DEEP2 (Gerke et al. 2007; Cooper et al. 2007, 2008), VVDS (Cucciati et al. 2006), COSMOS (Cassata et al. 2007), and SHADES (Serjeant et al. 2008).

A complementary approach to measuring the environmental dependence of star formation has been the detailed study of galaxy clusters. This method ensures the inclusion of large numbers of galaxies in high-density environments, which may be rare in field surveys. Furthermore, cluster studies can help clarify how the global cluster environment influences galaxy properties relative to the local galaxy environment. The SFRs in clusters have been examined in various studies, including surveys of multiple clusters such as CNOC (Balogh et al. 1998, 1999; Balogh \& Morris 2000; Ellingson et al. 2001), MORPHS (Dressler et al. 1999; Poggianti et al. 1999), EDisCS (Poggianti et al. 2006, 2008; Finn et al. 2005), the Spitzer/MIPS GTO team (Bai et al. 2007; Marcillac et al. 2007), and SMIRCS (Saintonge et al. 2008; Tran et al. 2009), as well as studies of individual clusters (e.g., Finn et al. 2004; Kodama et al. 2004; Geach et al. 2006).

A full understanding of the evolution of the environmental dependence of SFR has been impeded by at least two observational limitations. First, most of the above general field and targeted cluster studies were carried out in the rest-frame optical, which suffers from the effects of dust extinction. Because 
Table 1

Summary of $24 \mu \mathrm{m}$ Detections

\begin{tabular}{|c|c|c|c|c|c|c|c|}
\hline Cluster & $z$ & $\begin{array}{c}\sigma \\
\left(\mathrm{km} \mathrm{s}^{-1}\right) \\
\end{array}$ & $\begin{array}{l}f_{\min }{ }^{\mathrm{a}} \\
(\mu \mathrm{Jy})\end{array}$ & $\begin{array}{l}f_{\max }^{\mathrm{b}} \\
(\mu \mathrm{Jy})\end{array}$ & $\begin{array}{c}f_{80}^{\mathrm{c}} \\
(\mu \mathrm{Jy})\end{array}$ & $\begin{array}{c}L_{\mathrm{IR}}(80)^{\mathrm{d}} \\
\log _{10}\left(L_{\mathrm{IR}} / L_{\odot}\right)\end{array}$ & $\begin{array}{c}\mathrm{SFR}_{80}{ }^{\mathrm{e}} \\
\left(M_{\odot} \mathrm{yr}^{-1}\right)\end{array}$ \\
\hline CL1216.8 - 1201 & 0.7943 & $1018_{-77}^{+73}$ & 13 & 2347 & $75 \pm 5$ & 10.91 & 13.9 \\
\hline CL1354.2 - 1230 & 0.7620 & $648_{-110}^{+105}$ & 3 & 2856 & $82 \pm 3$ & 10.91 & 13.9 \\
\hline CL1054.7 - 1245 & 0.7498 & $504_{-65}^{+113}$ & 9 & 2599 & $75 \pm 3$ & 10.85 & 12.3 \\
\hline CL1040.7 - 1155 & 0.7043 & $418_{-46}^{+55}$ & 39 & 1296 & $80 \pm 3$ & 10.83 & 11.6 \\
\hline CL1054.4 - 1146 & 0.6972 & $589_{-70}^{+78}$ & 36 & 1068 & $72 \pm 3$ & 10.78 & 10.3 \\
\hline CL1227.9 - 1138 & 0.6357 & $574_{-75}^{+72}$ & 6 & 1517 & $80 \pm 3$ & 10.75 & 9.6 \\
\hline CL1353.0 - 1137 & 0.5882 & $666_{-139}^{+136}$ & 50 & 4072 & $100 \pm 3$ & 10.75 & 9.7 \\
\hline CL1037.9 - 1243 & 0.5783 & $319_{-52}^{+53}$ & 40 & 1556 & $82 \pm 3$ & 10.65 & 7.7 \\
\hline CL1232.5 - 1250 & 0.5414 & $1080_{-89}^{+119}$ & 43 & 1541 & $97 \pm 3$ & 10.64 & 7.5 \\
\hline CL1411.1 - 1148 & 0.5195 & $710_{-133}^{+125}$ & 49 & 3694 & $97 \pm 3$ & 10.59 & 6.6 \\
\hline CL1420.3 - 1236 & 0.4962 & $218_{-50}^{+43}$ & 44 & 1612 & $95 \pm 5$ & 10.52 & 5.7 \\
\hline CL1301.7 - 1139 & 0.4828 & $687_{-86}^{+81}$ & 42 & 1181 & $85 \pm 3$ & 10.43 & 4.6 \\
\hline CL1138.2 - 1133 & 0.4796 & $732_{-76}^{+72}$ & 43 & 1441 & $85 \pm 3$ & 10.42 & 4.6 \\
\hline CL1018.8 - 1211 & 0.4734 & $486_{-63}^{+59}$ & 37 & 5146 & $80 \pm 3$ & 10.38 & 4.1 \\
\hline CL1059.2 - 1253 & 0.4564 & $510_{-56}^{+52}$ & 41 & 5485 & $82 \pm 3$ & 10.35 & 3.9 \\
\hline CL1202.7 - 1224 & 0.4240 & $518_{-104}^{+92}$ & 22 & 5196 & $97 \pm 3$ & 10.33 & 3.6 \\
\hline
\end{tabular}

Notes.

a Minimum $24 \mu \mathrm{m}$ flux detected from sources with $\mathrm{S} / \mathrm{N}>2.5$.

b Maximum $24 \mu \mathrm{m}$ flux detected from sources with $\mathrm{S} / \mathrm{N}>2.5$.

c $24 \mu \mathrm{m}$ flux corresponding to $80 \%$ completeness limit.

${ }^{\mathrm{d}} L_{\mathrm{IR}}$ corresponding to $80 \%$ completeness limit. Relative error is the same as for $f_{80}$.

e SFR corresponding to $80 \%$ completeness limit. Relative error is the same as for $f_{80}$.

they make use of SFRs that are computed from long-wavelength data that are less affected by dust, the GOODS and SHADES studies are notable exceptions among the above general field studies, as are the Spitzer/MIPS GTO and SMIRC studies of clusters. Second, the total number of clusters studied remains fairly small in the face of the large cluster-to-cluster variations observed (e.g., Finn et al. 2005).

This paper is one in a series based on the ESO Distant Cluster Survey (EDisCS; White et al. 2005; Halliday et al. 2004; Poggianti et al. 2006; Desai et al. 2007). We address the above limitations by presenting Spitzer/MIPS observations of 16 intermediate-redshift $(0.42<z<0.8)$ EDisCS clusters. Not only does this triple the number of well-studied clusters at these redshifts, but the Multiband Imaging Photometer for Spitzer (MIPS) observations allow us to characterize the dustobscured SFRs. These observations also have the advantage that they provide us with a SFR-limited sample of cluster galaxies.

This paper is organized as follows. We describe the cluster sample in Section 2 and the Spitzer/MIPS data in Section 3. We then describe the selection of the cluster members in Section 4 and the conversion from observed $24 \mu \mathrm{m}$ flux to total IR luminosity in Section 5. In Section 6, we describe the sample we use for a field comparison. We present our results in Section 7, including the spatial and luminosity distributions of MIPS galaxies. In Section 8, we discuss our results in the context of cluster evolution scenarios, and we present our conclusions in Section 9.

We adopt a $\Lambda$ CDM cosmology, assuming $\Omega_{0}=0.3, \Omega_{\Lambda}=$ 0.7 , and $\mathrm{H}_{0}=70 \mathrm{~km} \mathrm{~s}^{-1} \mathrm{Mpc}^{-1}$ unless otherwise noted. All magnitudes are relative to Vega.

\section{ESO DISTANT CLUSTER SURVEY}

The clusters targeted in this survey are drawn from EDisCS (White et al. 2005). EDisCS is an ESO Large Programme that targeted 20 fields with Very Large Telescope (VLT) imaging and spectroscopy, and New Technology Telescope (NTT) nearIR imaging. In those fields, 26 structures (groups and clusters) have been identified (Halliday et al. 2004; Milvang-Jensen et al. 2008). One field contained no significant structure, and the structure cl1103.7-1245 at $z=0.96$ does not contain many spectroscopically confirmed members. The remaining 18 fields contain 17 primary structures and 7 secondary ones, where primary denotes the most massive structure in each field. These comprise the only sizable sample of low-mass clusters that has been studied in such detail at high redshift. Mass estimates for the EDisCS clusters have been derived from weak lensing (Clowe et al. 2006) and velocity dispersions.

We targeted only 16 primary clusters with Spitzer (we omit the primary structure in the cl1119.3-1129 field because of its low velocity dispersion). The redshift and velocity dispersions of the clusters are listed in Table 1 . All 16 clusters have extensive ground-based data that cover the same approximate area imaged by Spitzer, including multiband photometry and spectroscopy. There are 30-50 spectroscopically confirmed members per cluster. Photometric redshifts, spectroscopic results, and ground-based morphologies are available for a $5^{\prime} \times 5^{\prime}$ region around each cluster. Furthermore, derived-data products such as $k$-corrected absolute magnitudes and estimates of the total number of member galaxies (Pello et al. 2009; Rudnick et al. 2009) are readily available. The velocity dispersions and redshifts of the EDisCS clusters are not correlated (see Table 1), an important point to demonstrate before looking for evolutionary trends within the sample.

\section{SPITZER/MIPS DATA}

Infrared observations of cluster galaxies by the Infrared Space Observatory (ISO; Kessler et al. 1996) allowed the first look at obscured star-forming galaxies in distant clusters 
(Metcalfe et al. 2005). However, systematic coverage of clusters could not be conducted with $I S O$ given the time it would have required to obtain multi-positioned and heavily overlapped rasters of such targets. The Spitzer Space Telescope (Werner et al. 2004) provides not only the desired coverage of clusters, but with the improved sensitivity necessary to probe the infrared properties of cluster galaxies to lower masses and to larger redshifts, providing a unique opportunity to explore their evolution.

\subsection{Observations}

We obtained images of the clusters at $24 \mu \mathrm{m}$ using the Multiband Imaging Photometer for Spitzer (MIPS; Rieke et al. 2004). Observations of the 16 clusters were taken during Cycles 2 and 3 under guest observer programs 20009 and 30102. We imaged the central $5^{\prime} \times 5^{\prime}$ of each cluster to match the areal coverage of the ground-based VLT data. This area corresponds to a projected size of $1.8 \times 1.8 \mathrm{Mpc}$ at $z=0.5$ and $2.3 \times 2.3$ $\mathrm{Mpc}$ at $z=0.8$, which incorporates $>90 \%$ of the volume within the virial region at both epochs, assuming a typical comoving virial radius of $1 \mathrm{Mpc}$.

To map the $5^{\prime} \times 5^{\prime}$ area, we use MIPS Photometry in large field mode. We complete 10 cycles of $10 \mathrm{~s}$ exposures for the $0.42<z<0.52$ clusters and 20 cycles of $10 \mathrm{~s}$ exposures for the higher-redshift clusters. We use a $20^{\prime \prime}$ sky offset, which effectively doubles our on-source exposure time.

\subsection{Data Reduction}

The post-basic calibration data (BCD) images produced by the Spitzer Science Center (SSC) pipeline show large-scale variations in the sky level that are not effectively corrected by the flat field. We therefore start our data reduction by applying an additional flat-field correction to the BCD images. To do this, we first run SExtractor (Bertin \& Arnouts 1996) on individual BCD images. Using the source list from SExtractor, we mask all pixels that lie within a 10 pixel radius of an object. We then average the masked $\mathrm{BCD}$ images using the IRAF routine imcombine to create a flat image.

We use the MOsaicker and Point source EXtractor $\left(\mathrm{MOPEX}^{14}\right)$ routine mosaic.pl to create a mosaic of the flattened images, and we fit the point response function (PRF) in each mosaiced image using the Astronomical Point Source EXtraction (APEX) routine prf_estimate.pl. We then use the APEX routine apex_lframe.pl to identify sources and extract aperture photometry. We use the total flux value calculated by MOPEX.

To test for systematics in our data reduction procedure, we compare the aperture fluxes from the mosaic made from MOPEX with a mosaic made using the MIPS Instrument Team's Data Analysis Tool version 3.06 (DAT; Gordon et al. 2005) for the cluster CL1216. The average ratio of the DAT to MOPEX aperture fluxes measured in an aperture with a 4 pixel (10!2) diameter is $1.02 \pm 0.09$, so fluxes are consistent at the $10 \%$ level. The rms increases with aperture size; the average ratio is $1.05 \pm 0.18$ using an aperture with a diameter of 6 pixels (15".3). Fluxes resulting from MOPEX and DAT reductions are consistent, and we use the MOPEX reduction for the rest of the sample.

\footnotetext{
${ }^{14}$ APEX was written for the SSC by David Makovoz.
}

\subsection{Completeness}

We use the IRAF ${ }^{15}$ artdata package to estimate our detection efficiency as a function of source brightness. The cluster galaxies that we are studying are smaller than the MIPS $24 \mu \mathrm{m}$ pointspread function (PSF), so we add point sources into the final mosaiced image. We add 1000 sources with fluxes ranging uniformly from $10 \mu \mathrm{Jy}$ to $180 \mu \mathrm{Jy}$. The artificial sources are positioned randomly on each mosaiced image, avoiding edges and previously placed artificial sources. We limit the number of artificial sources to 10 per image so that we do not significantly alter the source density and repeat the simulation 100 times per cluster image to accumulate 1000 artificial sources per cluster. We convolve the sources with a PRF from the SSC derived from a mosaiced $24 \mu \mathrm{m}$ image. We use this rather than the PRF measured from each image because our measured PRFs are frequently contaminated by nearby sources whereas the SSC PRF is not. We then rerun apex_lframe.pl and determine the fraction of artificial sources detected as a function of source brightness. The results show that our $80 \%$ completeness limit ranges from $72 \mu \mathrm{Jy}$ to $100 \mu \mathrm{Jy}$, with the $80 \%$ completeness flux level listed for individual clusters in Table 1.

\section{ASSEMBLY OF CLUSTER SAMPLES}

\subsection{Optical Counterparts of $24 \mu \mathrm{m}$ Sources}

Although the alignment of the optical and $24 \mu \mathrm{m}$ images is good, we find systematic offsets between the images that can be as large as $1^{\prime \prime} 1$. To correct for this misalignment, we perform a first-pass match between the optical and IR sources. From the matched sources, we calculate the average offset between the optical and $24 \mu \mathrm{m}$ positions and then adjust the $24 \mu \mathrm{m}$ coordinates so that the average offset between the optical and IR positions is zero in both R.A. and decl. We then rematch the optical and IR sources using the shifted $24 \mu \mathrm{m}$ coordinates.

We find a total of $233724 \mu \mathrm{m}$ sources in the 16 cluster images that have a signal-to-noise ratio $(\mathrm{S} / \mathrm{N})$ greater than 2.5. We are able to match 1911 of these $(82 \% \pm 1 \%)$ to optical counterparts in the EDisCS source catalogs using a match radius of $2^{\prime \prime}$ between the $24 \mu \mathrm{m}$ and optical source. When multiple sources lie within $2^{\prime \prime}$, we select the optical counterpart that is closest to the $24 \mu \mathrm{m}$ source. Overall, 222 sources (9\%) have more than one optical match within $2^{\prime \prime}$.

We find $42624 \mu \mathrm{m}$ detections that are not matched to an object in the EDisCS catalog. Some of these IR sources (79) are false detections, lying near the edge of the $24 \mu \mathrm{m}$ image or associated with the Airy ring of a bright $24 \mu \mathrm{m}$ source. Other IR sources (48) are unmatched because they overlap a bright, extended optical source, and thus any optical counterpart is undetectable in the optical image. The number of remaining unmatched $24 \mu \mathrm{m}$ sources is 268 . Of these, 125 sources $(5 \%$ of the IR galaxies) appear to be a blend of one or more optical sources; these have an optical counterpart, it is just not clear which one is the counterpart. We consider the remaining 143 sources to be obscured sources; 76 coincide with a faint optical source that is below the detection limit of the EDisCS catalog, and the remaining 67 sources have no optical counterpart in the EDisCS I-band image. These sources are likely to lie at redshifts beyond our clusters (e.g., Le Floc'h et al. 2005).

\footnotetext{
15 IRAF is distributed by the National Optical Astronomy Observatory, which is operated by the Association of Universities for Research in Astronomy (AURA) under cooperative agreement with the National Science Foundation.
} 


\subsection{Selection of Cluster Members}

We calculate cluster membership in two complementary ways. We use spectroscopic redshifts to conclusively establish the membership when available. The majority of galaxies in each cluster, however, does not have spectroscopy, and we therefore use photometric redshifts to determine cluster membership for the galaxies with no spectroscopy. The EDisCS spectroscopy is described in detail in Halliday et al. (2004) and Milvang-Jensen et al. (2008), and the photometric membership techniques are described in detail in Pello et al. (2009) and Rudnick et al. (2009). Here we provide a brief summary of the techniques used in this paper.

Spectroscopic members are those with velocities within $\pm 3 \sigma$ from the cluster redshift. Spectroscopic membership information supersedes all photometric information. The photometric redshifts have been computed from the full optical/NIR photometry and have been calibrated from the extensive EDisCS spectroscopy to cull non-members, while retaining $>90 \%$ of all confirmed cluster members independent of rest-frame color, down to the spectroscopic magnitude selection limit, which was $22<I<23$ depending on the cluster. The photometric redshifts only yield robust membership classifications when optical and NIR data are present, and so we limit our sample to those areas of each field with adequate exposure in all bands.

To test the reliability of our photometric redshifts for the IRselected galaxies in our sample, we compare the completeness and the contamination of the photometric membership for IR and non-IR detected galaxies using our extensive spectroscopy. We define the completeness as the number of spectroscopic members that are also photometric redshift members, divided by the number of spectroscopic members. We find a completeness of $85 \% \pm 6 \%$. For the subsample of spectroscopic members that are also $24 \mu \mathrm{m}$ sources, we find a completeness of $85 \% \pm 12 \%$. We define contamination as the number of spectroscopic nonmembers that are classified as photometric members, divided by the total number of spectroscopic members that are classified as photometric members. This yields a contamination of $48 \% \pm 3 \%$. For the subsample of spectroscopic members that are also $24 \mu \mathrm{m}$ sources, we find a contamination of $53 \% \pm 6 \%$. Thus, the completeness and contamination of the $24 \mu \mathrm{m}$ sources are entirely consistent with the optically selected cluster sample down to the spectroscopic magnitude limit. Marcillac et al. (2007) also find that the accuracy of photometric redshifts for IR-selected galaxies in a $z=0.83$ cluster is the same as that for all cluster members.

\section{TOTAL INFRARED LUMINOSITY}

We use the Dale \& Helou (2002) models to estimate total IR luminosity $(3-1100 \mu \mathrm{m})$ from $24 \mu \mathrm{m}$ fluxes as a function of galaxy redshift. To first calculate the $24 \mu \mathrm{m}$ luminosity, we multiply the $24 \mu \mathrm{m}$ flux (in Jy) by $4 \pi d_{L}^{2}$, where $d_{L}$ is the luminosity distance corresponding to the cluster redshift, and by $c / 23.8 \mu \mathrm{m}$, the central frequency of the $24 \mu \mathrm{m}$ bandpass. We scale the $24 \mu \mathrm{m}$ luminosity by the conversion found from the Dale \& Helou (2002) templates corresponding to the cluster redshift to estimate $L_{\mathrm{IR}}$. According to the Dale \& Helou (2002) templates, the error associated with estimating the IR luminosity solely from the observed $24 \mu \mathrm{m}$ flux varies with redshift; for the redshift range spanned by the EDisCS clusters, the error ranges from a minimum of $5 \%$ at $z=0.6$ to a maximum of $22 \%$ at $z=0.8$. Finally, we divide $L_{\mathrm{IR}}$ by the luminosity of the Sun, where $L_{\odot}=3.826 \times 10^{33} \mathrm{erg} \mathrm{s}^{-1}$.
Active galactic nuclei (AGNs) will contaminate our measurements with flux that is not associated with star formation. Mid-infrared colors from the Infrared Array Camera (IRAC; Fazio et al. 2004), spanning 3-8 $\mu \mathrm{m}$, will help identify AGNs (Stern et al. 2005; Donley et al. 2007; Lacey et al. 2008) and will be presented in a future paper. However, Sánchez-Blázquez et al. (2009) analyze the optical spectra of EDisCS galaxies and find that most of the emission-line galaxies are powered by star formation rather than AGNs. Furthermore, Bell et al. (2005) estimate that $\lesssim 15 \%$ of the total infrared luminosity density at $0.65<z<0.75$ is from sources with significant AGN emission, and the contribution is likely much lower since the infrared luminosity in these galaxies may also arise from star formation (see also Robaina et al. 2009 for a more detailed discussion). Similarly, the Bai et al. (2007) and Marcillac et al. (2007) studies of clusters at similar redshifts to our sample find that only $\sim 4$ out of 66 IR-detected galaxies are unambiguously AGNs and argue that the rest of their galaxies are dominated by dusty starbursts. Finally, Geach et al. (2009) observe a sample of 12 galaxies that are members of a $z=0.4$ cluster with the Infrared Spectrograph (IRS; Houck et al. 2004). The results of the IRS analysis indicate that the mid-IR emission for 11 of 12 galaxies is powered by a starburst rather than AGN. Based on these results, we conclude that contamination by AGN is likely to be small.

We use the relation from Kennicutt (1998) to convert from $L_{\mathrm{IR}}(8-1000 \mu \mathrm{m})$ to SFR. There is a slight discrepancy between the definition of $L_{\mathrm{IR}}$ used by the Dale \& Helou (2002) models (3-1100 $\mu \mathrm{m})$ and that used in the Kennicutt star formation conversion $(8-1000 \mu \mathrm{m})$, but this impacts the inferred SFR by less than $5 \%$ (D. Dale 2009, private communication).

When we translate the $80 \%$ completeness flux limits listed in Table 1 to IR luminosities, we find that we do not probe as deeply in the higher-redshift clusters. CL1216 and CL1353 have the highest $80 \%$ completeness luminosities of $\log _{10}\left(L_{\mathrm{IR}} / L_{\odot}\right)=10.91$, which corresponds to an IR-derived SFR of $\sim 13 M_{\odot} \mathrm{yr}^{-1}$. This is slightly below the luminosity of luminous infrared galaxies (LIRGs; $\log _{10}\left(L_{\mathrm{IR}} / L_{\odot}\right)>11, L_{\mathrm{IR}}>$ $3.8 \times 10^{44} \mathrm{erg} \mathrm{s}^{-1}$; Sanders \& Mirabel 1996). Therefore, we are sampling the population of luminous infrared galaxies rather than normal star-forming galaxies uniformly across the redshift range. Because our $L_{\mathrm{IR}}$ limit is so close to the LIRG limit and given the uncertainties in computing $L_{\mathrm{IR}}$ solely from observed $24 \mu \mathrm{m}$ flux, we refer to our IR galaxies as LIRGs.

\section{GEMS FIELD SAMPLE}

Discrimination among cluster-specific processes that might affect the gas content of member galaxies requires a comparison between the star formation properties of cluster and field galaxies. The Galaxy Evolution from Morphology and SEDs (GEMS; Rix et al. 2004) project provides a good field sample to compare with our clusters because it is currently the largest sample of intermediate-redshift galaxies with accurate photometric redshifts and space-based morphologies. The GEMS survey covers an $\sim 800$ square arcminute region centered on the Extended Chandra Deep Field S (ECDF-S). The GEMS survey area was chosen to overlap with the Classifying Objects by Medium-Band Observations in 17 filters survey (COMBO-17; Wolf et al. 2001, 2004), which provides accurate photometric redshifts $\left(\sigma_{z} /(1+z) \sim 0.02\right)$ out to $z<1.2$. Most importantly for our purposes, the GEMS survey area was also imaged by the Spitzer MIPS GTO team at $24 \mu \mathrm{m}$ (Papovich et al. 2004) to a depth comparable to our imaging $(5 \sigma$ detection limit $=83 \mu \mathrm{Jy})$. 

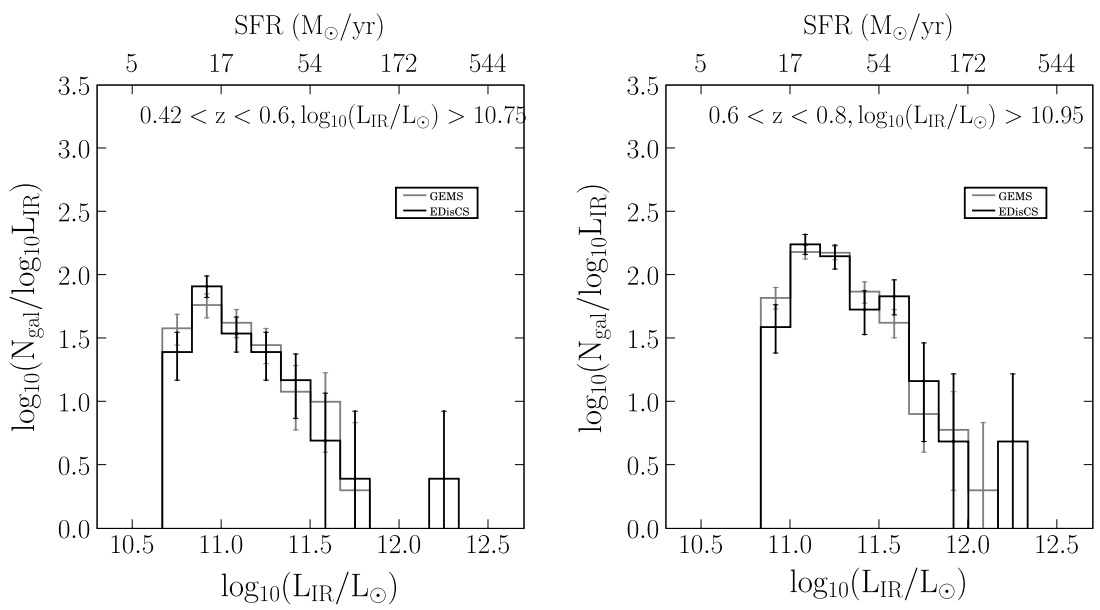

Figure 1. Distribution of $L_{\mathrm{IR}}$ for EDisCS (black) and GEMS (gray) samples for the $0.42<z<0.6$ sample (left) and $0.6<z<0.8$ sample (right). A lower IR luminosity cut is used for the lower-redshift sample, but in each redshift bin, the same $L_{\mathrm{IR}}$ and magnitude cut is applied to the EDisCS and GEMS galaxies. The EDisCS histograms are scaled to match the total number of GEMS galaxies. The error bars show Poisson errors. The IR luminosities of the field and clusters galaxies are indistinguishable for both low- and high- $z$ samples, indicating that the $L_{\mathrm{IR}}$ distribution of the most actively star-forming galaxies is not affected by the cluster environment. The top horizontal axes show IR-derived SFRs.

The IR properties of the GEMS galaxies are studied in detail by Bell et al. (2005) and Le Floc'h et al. (2005).

To build a comparison sample for our clusters, we select all GEMS galaxies within $0.42<z<0.8, I<24$, and that lie within the $24 \mu \mathrm{m}$ imaging area. In addition, we require an $\mathrm{S} / \mathrm{N}$ of at least 3 in both $I$ and $V$ observed magnitudes. We compute $L_{\mathrm{IR}}$ from the observed $24 \mu \mathrm{m}$ flux using the redshift-dependent conversion described in Section 5 rather than using the $L_{\mathrm{IR}}$ values of Bell et al. (2005). When comparing with EDisCS galaxies, we impose the following $L_{\mathrm{IR}}$ cuts: $\log _{10}\left(L_{\mathrm{IR}} / L_{\odot}\right)>10.75$ for the $0.42<z<0.6$ galaxies, and $\log _{10}\left(L_{\mathrm{IR}} / L_{\odot}\right)>10.95$ for the $0.6<z<0.8$ galaxies. The $L_{\mathrm{IR}}$ limit for the low- $z$ sample is set by the depth of the low- $z$ EDisCS imaging, whereas the $L_{\mathrm{IR}}$ limit for the high- $z$ sample is set by the depth of the GEMS imaging.

\section{ANALYSIS AND RESULTS}

\subsection{Properties of IR Galaxies}

\subsubsection{IR Luminosity Distribution}

We compare the $L_{\mathrm{IR}}$ distribution of our full sample with that of the GEMS field sample in Figure 1. We split both the field and cluster samples at $z=0.6$ to minimize evolutionary effects. The $z<0.6$ sample includes 87 GEMS galaxies and 75 EDisCS galaxies, and the $z>0.6$ sample includes 250 GEMS galaxies and 102 EDisCS galaxies. We show the luminosity distribution in terms of $N_{\mathrm{gal}} / \log _{10} L_{\mathrm{IR}}$ in Figure 1 for the lower- (left) and higher-redshift (right) samples. After scaling the EDisCS distribution and errors to adjust for the difference in sample size, the GEMS and EDisCS distributions agree within errors for both the low and high-redshift samples. Furthermore, a two-sided Kolmogorov-Smirnoff (K-S) test cannot distinguish the cluster and field distributions in both redshift bins. Thus, the IR luminosity distribution of the most active star-forming galaxies is not affected by the cluster environment.

\subsubsection{Colors of IR Galaxies}

The location of the $24 \mu \mathrm{m}$ sources on the cluster color-magnitude diagrams is shown in Figure 2. The solid black line in each panel is the fit to the red sequence from De Lucia et al. (2007), who assume a fixed red-sequence slope of -0.09 and fit the zero point to the non-emission line spectroscopically confirmed cluster members. The dashed lines mark a color offset of $\Delta(V-I)<0.3$ from the red sequence, illustrating the selection criteria for red sequence members used here and in previous analyses of the EDisCS clusters (De Lucia et al. 2007; Sánchez-Blázquez et al. 2009; Rudnick et al. 2009). We define blue cluster galaxies as those with $V-I$ colors at least $0.3 \mathrm{mag}$ bluer than the red sequence. One main result from Figure 2 is that $21_{-4}^{+6} \%(16 / 77)$ of the spectroscopically confirmed cluster LIRGs lie on the red sequence while the remaining $79_{-6}^{+4} \%$ (61/ 77) lie in the blue cloud. This fraction of red LIRGs is higher than that observed by Tran et al. (2009) in a $z=0.35$ cluster and is comparable to the fraction they measure in the field. Tran et al. (2009) use a $B-V$ cut to select red galaxies rather than the $V-I$ cut used in this paper, and this might account for some of the discrepancy.

Figure 2 shows that there is not a one-to-one correspondence between [O II]-emitting (blue circles) and IR-bright galaxies (red circles). Only a minority $(29 \% \pm 3 \%)$ of spectroscopic members with [O II] emission (EW(O II) $>5 \AA$ ) are detected at $24 \mu \mathrm{m}$. Conversely, $83_{-5}^{+4} \%(64 / 77)$ of the IR galaxies show $\left[\mathrm{O}_{\mathrm{II}}\right]$ emission in their optical spectra $\left(\mathrm{EW}\left(\left[\mathrm{O}_{\mathrm{II}}\right]\right)>5 \AA\right)$. The remaining IR galaxies have weaker emission lines and lie predominantly on the red sequence, consistent with the results of Sánchez-Blázquez et al. (2009). The 13 IR sources with no [O II] emission do not dominate the total $L_{\mathrm{IR}}$ but contribute a fraction that is entirely consistent with their number. Thus, we do not find a large fraction of optically selected cluster members whose star formation is completely obscured at visible wavelengths. However, the IR-derived SFRs greatly exceed those derived from dust-corrected [O II] emission for the majority of the IR galaxies; the median ratio of SFR(IR)/SFR(O II) is 2.9 for the Spitzer-detected galaxies in the EDisCS clusters (Vulcani et al. 2010).

Of particular interest are the $24 \mu \mathrm{m}$ sources on the red sequence. One possibility is that they are ellipticals with AGN rather than star-forming galaxies. Of the red-sequence galaxies for which we have Hubble Space Telescope (HST) morphologies, six have IR emission: one is an elliptical and the remaining five are normal spirals. Thus, the majority of the reddest IR sources appear to be red because of dust, and future 


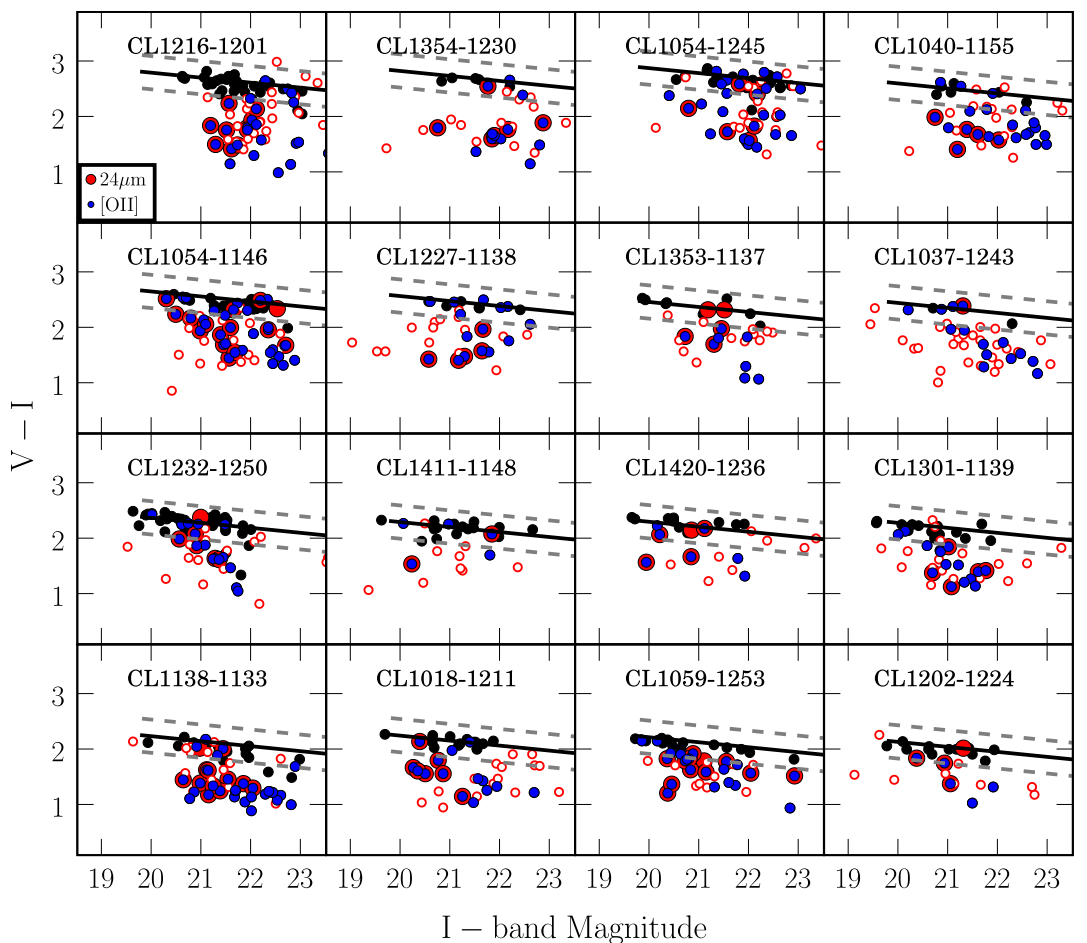

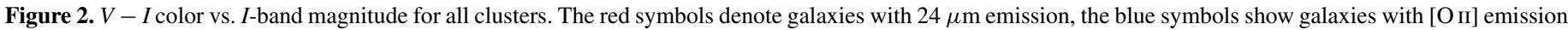

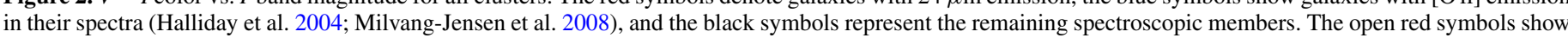

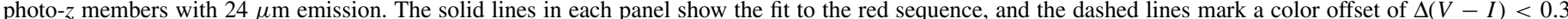

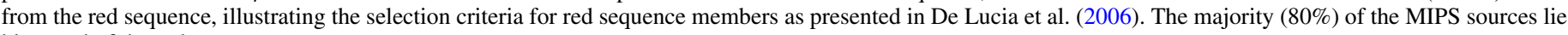
blueward of the red sequence.

analysis will probe the properties of members on or near the red sequence, including AGN emission, in more detail.

\subsubsection{Magnitudes of IR Galaxies}

In Figure 3, we show $L_{\mathrm{IR}}$ versus $M_{V}$ for individual clusters, with the panels ordered by decreasing redshift from left to right and top to bottom. The dotted line shows the $80 \%$ completeness limit for each cluster. The dashed line shows the 80\% completeness limit for CL1216, which has the highest threshold of all the clusters. The cluster-to-cluster variations in the distribution of $L_{\mathrm{IR}}$ are striking and illustrate the need for large samples of clusters to properly characterize star formation in dense environments at a given epoch. Furthermore, the higherredshift clusters appear to have more galaxies with high values of $L_{\mathrm{IR}}$. The redshift range of the EDisCS clusters corresponds to a time interval of 2.4 Gyr. Given the dramatic decline in SFRs since $z \sim 1$ as discussed in Section 1, one might expect to observe evolution within the EDisCS sample, and we examine this is more detail in Section 7.3.

In Figure 4, we combine the $0.42<z<0.6$ and $0.6<$ $z<0.8$ cluster samples to show IR luminosity versus restframe absolute $V$ magnitude as a function of galaxy color. The corresponding GEMS samples are shown with gray circles. Both field and cluster samples are complete in the top right quadrant of each plot, at $M_{V}>-19$ (set by the depth of the GEMS survey) and $\log _{10}\left(L_{\mathrm{IR}}\right)>\log _{10}\left(L_{\mathrm{lim}}\right)$, where $\log _{10}\left(L_{\mathrm{lim}}\right)=10.95$ for the high- $z$ samples and 10.75 for the lower- $z$ samples. For the EDisCS clusters, we separate the blue and red galaxies as described in Section 7.1.2. To separate red and blue GEMS galaxies in an analogous manner, we fit the red-sequence zero point of the EDisCS clusters as a function of cluster redshift. The resulting red sequence is given by:

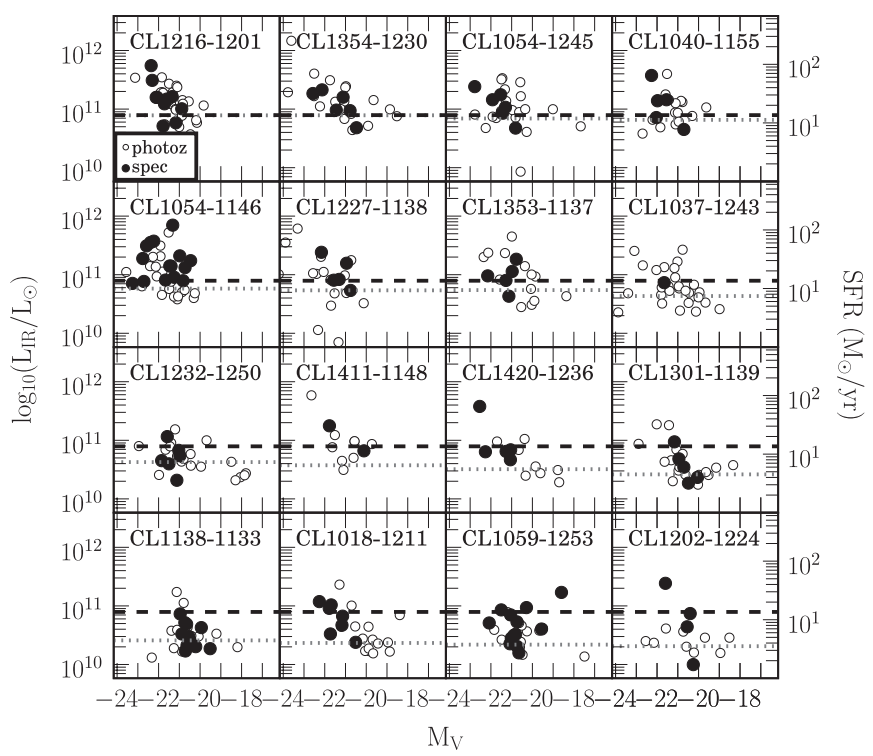

Figure 3. $L_{\mathrm{IR}}$ vs. $M_{V}$ for EDisCS clusters. The filled and open circles show the spectroscopic and photometric-redshift members, respectively. The dotted horizontal line shows the $80 \%$ completeness limit for each cluster. The dashed horizontal line shows $\log _{10}\left(L_{\mathrm{IR}}\right)=10.91$, the $80 \%$ completeness limit of CL1216. The cluster name is listed at the top of each panel, and the clusters are ordered by decreasing redshift from left to right and top to bottom. The $L_{\mathrm{IR}}$ of the $24 \mu \mathrm{m}$-emitting galaxies decreases with redshift. The right-hand vertical axes show IR-derived SFRs.

$-0.09(I-20)+1.9(z-0.42)+2.14$. As with the EDisCS galaxies, we define blue galaxies as those with $V-I$ colors at least 0.3 mag bluer than the red sequence. The main result from Figure 4 is that the $L_{\mathrm{IR}}-M_{V}$ distribution in all subplots are 


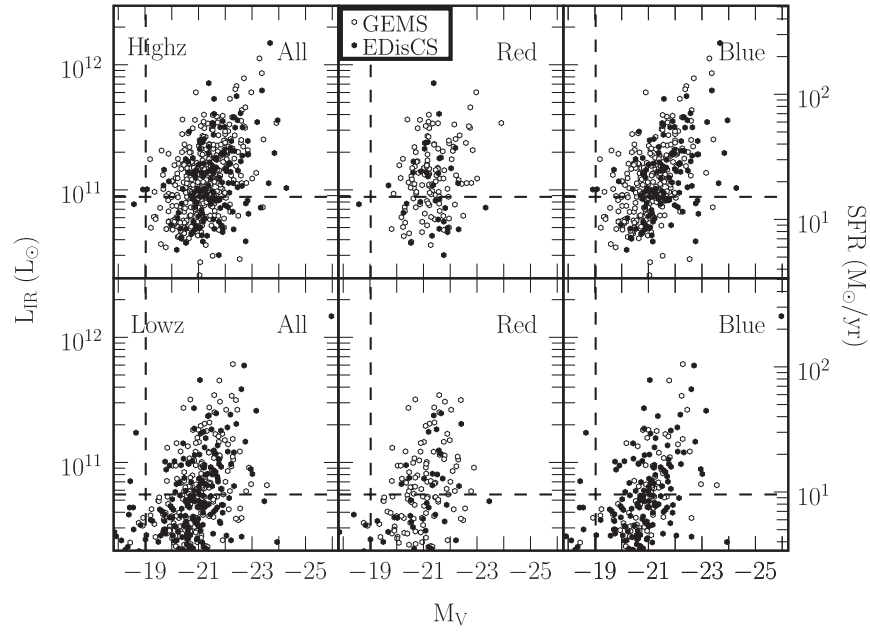

Figure 4. IR luminosity vs. rest-frame absolute $V$ magnitude. The top and bottom rows show the $0.6<z<0.8$ and $0.42<z<0.6$ EDisCS (black) and GEMS (white) galaxies, respectively. The columns show the $L_{\mathrm{IR}}-M_{V}$ distribution for all, red, and blue galaxies, respectively. The dashed horizontal lines show the IR completeness limit while the dashed vertical lines show the magnitude limit of $M_{V}<-19$. The high- $z$ blue cluster galaxies have systematically brighter luminosities than the high- $z$ blue field galaxies. The $L_{\mathrm{IR}}-M_{V}$ distribution of all other subgroups are consistent.

consistent except for the high- $z$ blue galaxies; the high- $z$ blue cluster galaxies have systematically brighter luminosities than the high- $z$ blue field galaxies.

\subsubsection{Stellar Masses of IR Galaxies}

In Figure 5, we show IR luminosity versus stellar mass for the higher- $z$ and lower- $z$ samples. Again, we show the blue and red galaxies separately. We calculate stellar mass using the relation from Bell et al. (2003):

$$
\log _{10}\left(M_{*} / L\right)_{B}=1.737(B-V)-0.942 .
$$

We adopt a stellar mass completeness limit of $\log _{10}\left(M_{*}\right)=$ 10.2 (vertical dashed line), which we calculate using the magnitude limit $M_{V}=-19$ and an assumed color of $B-V<1$.

A two-sided, two-sample K-S test indicates that the $L_{\mathrm{IR}}-M_{*}$ distribution of the higher- $z$ galaxies are significantly different (where we define significant as $>3 \sigma$ ). As shown in Figure 1, the IR luminosities of the field and cluster galaxies are consistent. The $L_{\mathrm{IR}}-M_{*}$ distributions differ because the stellar masses of the EDisCS red and blue IR galaxies are systematically higher than the stellar masses of the GEMS galaxies. In the lower- $z$ samples, only the $L_{\mathrm{IR}}-M_{*}$ distribution of the red galaxies differs significantly, and the sense of the difference is the same: the red EDisCS galaxies have higher stellar masses on average while their IR luminosities are consistent with the GEMS galaxies.

The results imply that the specific SFRs of all higher- $z$ cluster galaxies and the red lower- $z$ cluster galaxies are lower than the corresponding field galaxies. Given the uncertainties associated with comparing the colors and magnitudes from two different surveys, the significance of the difference in the $L_{\mathrm{IR}}-M_{*}$ distributions is difficult to assess. However, Vulcani et al. (2010) perform a similar analysis, comparing the spectroscopically confirmed EDisCS galaxies to field galaxies from the All-Wavelength Extended Groth Strip International Survey (AEGIS; Noeske et al. 2007). They measure SFRs from both [O II] emission and $24 \mu \mathrm{m}$ emission, and they find that both the lower and higher- $z$ cluster galaxies have lower

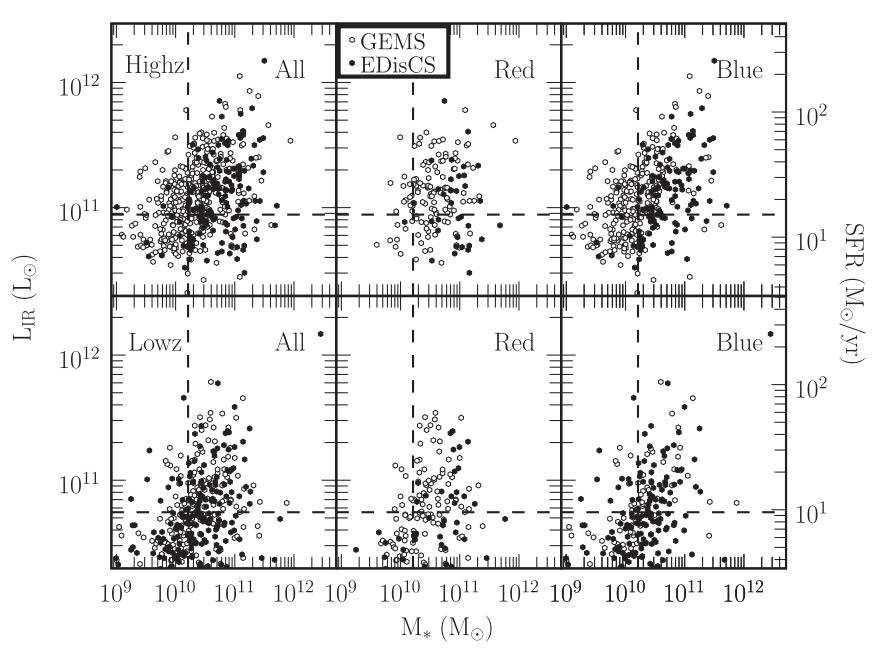

Figure 5. IR luminosity vs. stellar mass. The top and bottom rows show the $0.6<z<0.8$ and $0.42<z<0.6$ EDisCS (black) and GEMS (white) galaxies, respectively. The dashed horizontal lines show the IR completeness limit while the dashed vertical line shows the magnitude limit of $M_{V}<-19$. The EDisCS IR galaxies have higher stellar masses on average than the GEMS galaxies.

SFRs at a given stellar mass compared to the field. When comparing only blue galaxies, they find that the $z>0.6$ cluster galaxies have lower specific SFRs while the $z<0.6$ blue cluster galaxies do not. The difference becomes statistically significant in both redshift bins when red galaxies are included. In this paper, we find similar results using a different field sample and a different method of measuring SFRs. The addition of [O II] emission allows Vulcani et al. (2010) to probe to lower SFRs, but here we show that the difference between the field and cluster galaxies is detectable even among the most active star-forming galaxies. In addition, the differences that we measure are detected with higher significance according to a K-S test ( $>3 \sigma$ versus $2 \sigma$ ). This is likely due to the larger sample size used in this study which we obtain by including the photometric cluster members as well as the spectroscopic cluster members.

\subsection{Spatial Distribution of IR Galaxies}

To probe the spatial distribution of the IR galaxies, we calculate the fraction of $24 \mu \mathrm{m}$-emitting members as a function of projected radius from the cluster center. We again split the sample at $z=0.6$, and we use a lower $L_{\mathrm{IR}}$ cut of $\log _{10}\left(L_{\mathrm{IR}}\right)>10.75$ for the $z<0.6$ sample to improve statistics. We apply the same magnitude and $L_{\mathrm{IR}}$ cuts to the $z<0.6$ and $z>0.6$ GEMS galaxies. So while the results for the two epochs are not directly comparable, the cluster and field fractions in each epoch are.

We show the results for the $z<0.6$ and $z>0.6$ samples in the left and right panels of Figure 6, respectively. In both the lower and higher- $z$ panels, the fraction of IR cluster galaxies increases with projected radius and remains systematically lower than the field fraction over the area probed. The offset between the cluster and field is greater for the higher- $z$ clusters, but this may be due to the higher $L_{\mathrm{IR}}$ cut that is applied. The results are consistent with many other cluster studies (e.g., Balogh et al. 1997; Lewis et al. 2002; Gómez et al. 2003; Rines et al. 2005).

\subsection{Redshift Evolution of IR Activity}

To examine trends in the star-forming population with cluster redshift and mass, we characterize the star-forming activity 

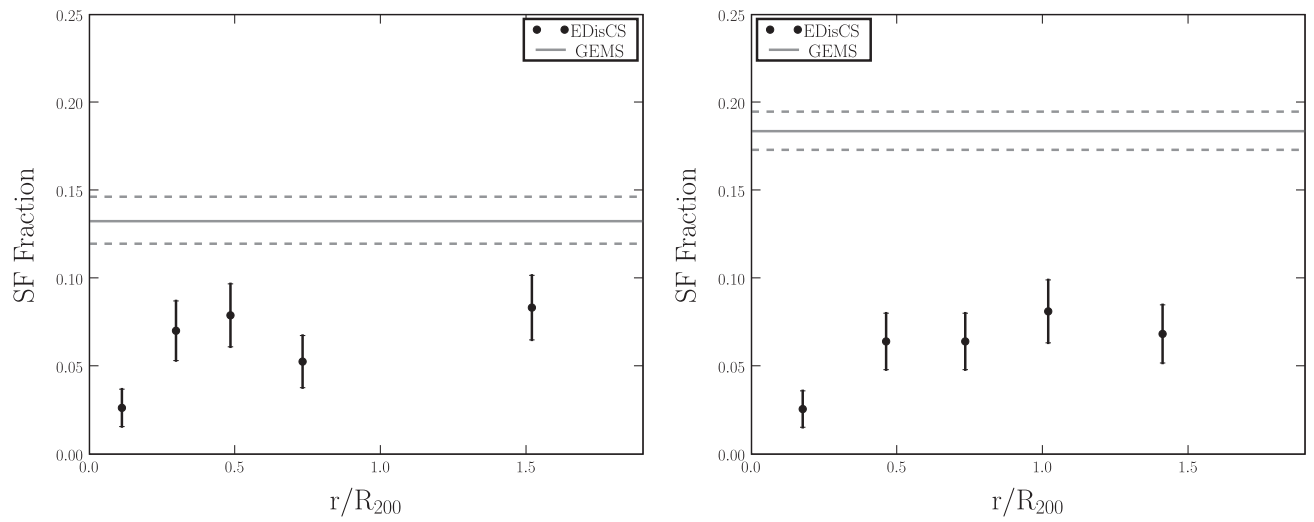

Figure 6. Left: fraction of IR galaxies $\left(\log _{10}\left(L_{\mathrm{IR}} / L_{\odot}\right)>10.75, M_{V}<-19\right)$ in equally populated bins as a function of projected separation from the cluster center for $z<0.6$ clusters only. Error bars show Poisson errors. The gray line shows the fraction of IR galaxies in the $0.42<z<0.6$ GEMS field sample, and the dashed lines show the $1 \sigma$ Poisson errors. The fraction of IR galaxies in the $z<0.6$ clusters increases with projected clustercentric radius out to $0.5 \times R_{200}$ and then levels. Right: fraction of IR galaxies $\left(\log _{10}\left(L_{\mathrm{IR}} / L_{\odot}\right)>10.95\right.$ and $\left.M_{V}<-19\right)$ in equally populated bins as a function of projected separation from the cluster center for $z>0.6$ clusters only. The gray line shows the fraction of IR galaxies in the $0.6<z<0.8$ GEMS field sample, and the dashed lines show the $1 \sigma$ Poisson errors. The fraction of IR galaxies increases with projected clustercentric radius out to $0.5 \times R_{200}$ and then levels.

of the clusters in three ways: the fraction of $24 \mu$ m-emitting galaxies, the average $L_{\mathrm{IR}}$ of the $24 \mu \mathrm{m}$ galaxies, and the total $L_{\mathrm{IR}}$ averaged over all cluster members. These quantities are shown in the top, middle, and bottom panels of Figure 7, respectively, versus cluster redshift (left) and velocity dispersion (right). We calculate the number of IR galaxies and the total IR luminosity per cluster using only those galaxies with $M_{V}<-19$ and $\log _{10}\left(L_{\mathrm{IR}} / L_{\odot}\right)>10.95$, and we normalize by the total number of cluster galaxies with $M_{V}<-19\left(N_{\text {tot }}\right)$. Note that we also calculate the various measures of star formation activity using an evolving $M_{V}$ limit as done in Poggianti et al. (2008). Specifically, we vary the $M_{V}$ cut from -20.5 at $z=0.8$ to -20.1 at $z=0.4$ to account for passive evolution. The results are not significantly impacted by the evolving magnitude cut, so we adopt a constant magnitude limit of $M_{V}<-19$.

We limit the analysis to galaxies that have a projected clustercentric radius less than $R_{200}$, where $R_{200}$ is the radius inside which the enclosed density is 200 times the critical density and approximates the virial radius of the cluster. The relationship between $R_{200}$ and velocity dispersion is shown in Equation (2) below, ${ }^{16}$

$$
R_{200}=2.47 \frac{\sigma_{x}}{1000 \mathrm{~km} \mathrm{~s}^{-1}} \frac{1}{\sqrt{\Omega_{\Lambda}+\Omega_{0}(1+z)^{3}}} h_{70}^{-1} \mathrm{Mpc},
$$

and we refer the reader to Finn et al. (2005) for a complete derivation of this relationship.

We show the three measures of star formation activity in Figure 7. The corresponding quantities for the GEMS galaxies (gray triangles and lines) are calculated using the same magnitude and $L_{\mathrm{IR}}$ cuts that were applied to the cluster galaxies. One main result from this figure is that the fraction of IR cluster galaxies lies significantly below that of the field, consistent with previous studies (e.g., Tran et al. 2009); the overall fraction of IR emitting galaxies is $6 \% \pm 1 \%$ for the cluster sample and $14 \% \pm 1 \%$ for the $0.42<z<0.8$ GEMS sample. Furthermore,

\footnotetext{
16 This derivation of $R_{200}$ assumes that the line-of-sight velocity dispersion is related to the circular velocity by $\sigma_{x}=v_{c} / \sqrt{3}$ rather than $\sigma_{x}=v_{c} / \sqrt{2}$ as one would expect if galaxies are orbiting isotropically in a single isothermal sphere (Finn et al. 2008). Nevertheless, we adopt this $\sqrt{3}$ scaling between $\sigma_{x}$ and $v_{c}$ for consistency with previous EDisCS publications and note that our value of $R_{200}$ is roughly $20 \%$ higher as a result.
}

all three measures of star formation activity show that the higherredshift clusters have a higher level of star formation activity than the lower-redshift clusters. The fraction of IR galaxies shows the most significant correlation $(\sim 3 \sigma)$ versus redshift as indicated by a Spearman rank test. The right panels of Figure 7 show that LIRG activity is not correlated with cluster velocity dispersion. Note that we are probing only the most actively starforming galaxies; Poggianti et al. (2006) find that star formation properties of less active galaxies do depend on cluster mass.

If we split the cluster sample into two redshift bins, we are able to detect redshift evolution with higher statistical significance. For example, the average fraction of LIRGs within $R_{200}$ is $9_{-1}^{+1} \%$ for the $z>0.6$ clusters and $4_{-1}^{+1} \%$ for the $z<0.6$ clusters. If we split the field sample at $z=0.6$, we find that the fraction of GEMS LIRGs drops from $18 \% \pm 1 \%$ to $8 \% \pm 1 \%$. The conclusion then is that the higher-redshift galaxies in all environments have a higher fraction of LIRGs and the amount of obscured star formation declines significantly over the 2.4 Gyr timeline spanned by the EDisCS clusters. In addition, the IR fraction declines by a factor of $2.25_{-0.65}^{+1.08}$ in the clusters and $2.25_{-0.36}^{+0.46}$ in the field. The cluster and field decline rates agree within the errors, and thus the rate at which the LIRG population declines is not affected by the cluster environment.

In Figure 8, we compare the redshift evolution of the LIRG fraction of the blue and red galaxies separately. To calculate the fractions, we divide the number of blue LIRGs by the total number of blue galaxies, and likewise for the red galaxies. The results for the blue galaxies are shown in the top panel. While a number of individual clusters (small circles) show a blue LIRG fraction that exceeds the field value, when the data are binned to improve statistics, the resulting LIRG fractions (large circles) among the blue cluster galaxies are consistent with the field values. The large cluster-to-cluster variations illustrate the need for large samples of clusters to accurately characterize star formation activity in cluster environments; observations of only one cluster might erroneously lead the observer to infer that clusters contain an excess of IR galaxies relative to the field. The evolution of the red LIRG fraction is shown in the bottom panel, and the red cluster galaxies lies systematically below that of the field. The difference between the cluster and field red LIRG fractions is large. The low fraction of red cluster LIRGs is due in part to the higher overall fraction of red galaxies in 


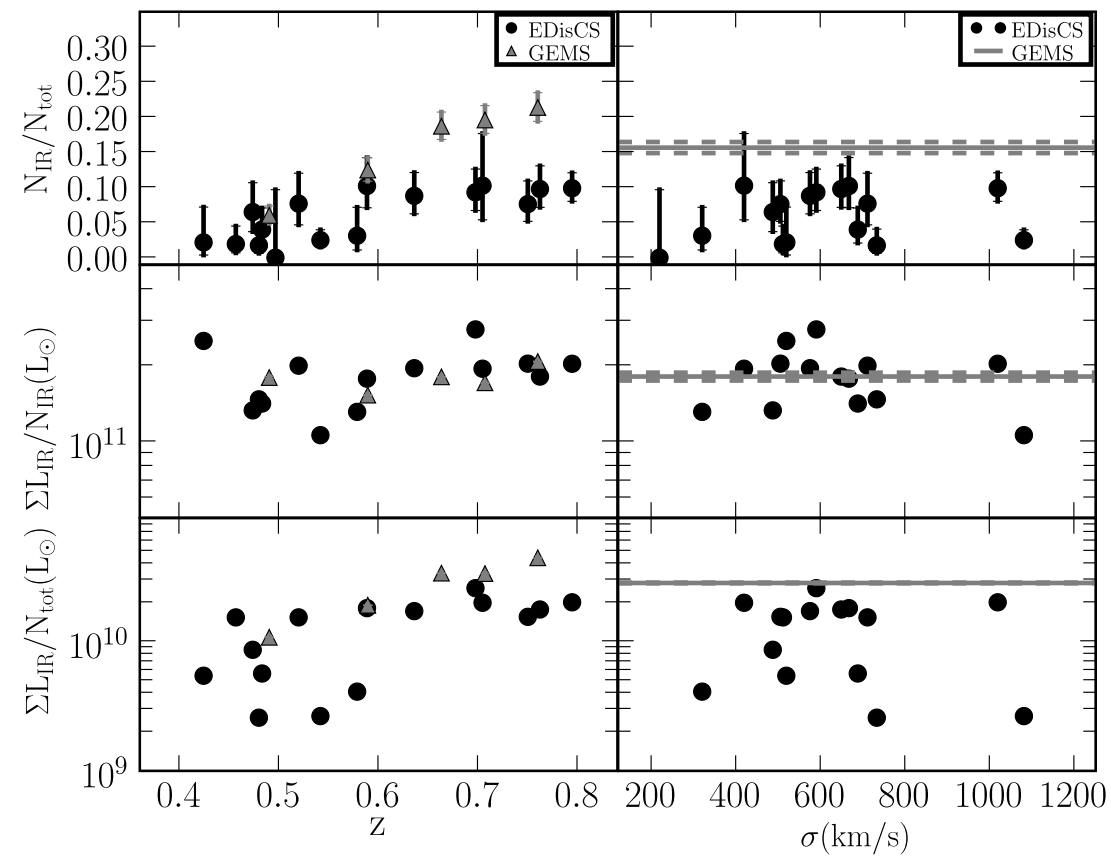

Figure 7. Fraction of IR galaxies $\left(\log _{10}\left(L_{\mathrm{IR}} / L_{\odot}\right)>10.95, M_{V}<-19 \mathrm{dr}<R_{200}\right)$ vs. cluster redshift (top left) and cluster velocity dispersion (top right). Total $L_{\mathrm{IR}}$ divided by the total number of IR-emitting members vs. cluster redshift (middle left) and cluster velocity dispersion (middle right). Total $L_{\mathrm{IR}}$ divided by the total number of members vs. cluster redshift (bottom left) and cluster velocity dispersion (bottom right). In all panels, the filled circles show quantities for the EDisCS clusters. The gray triangles (left panels) and gray lines (right panels) show the values for the GEMS field galaxies. Star formation activity is correlated with cluster redshift but not with cluster mass, and the star-formation activity of cluster galaxies is systematically lower than field galaxies. Cluster-to-cluster variations are large, illustrating the need for large samples.
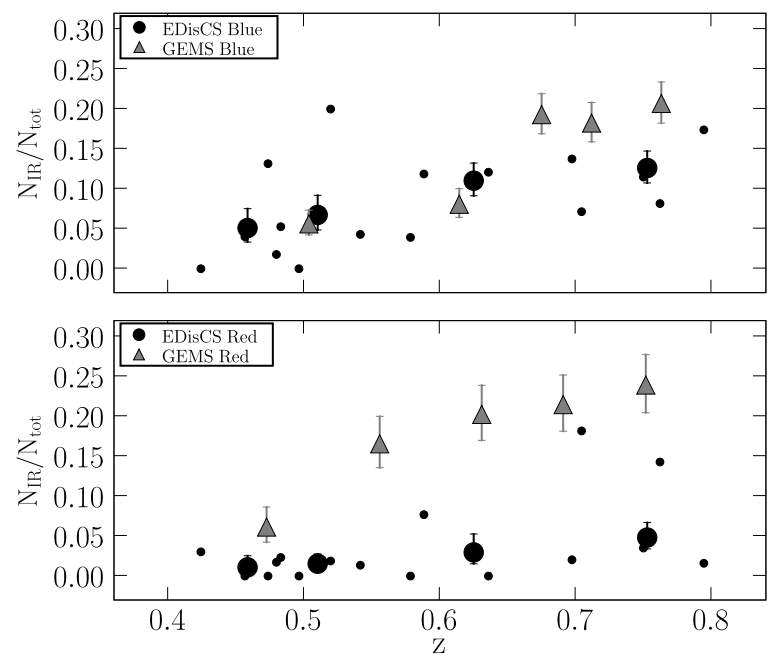

Figure 8. Fraction of IR galaxies $\left(\log _{10}\left(L_{\mathrm{IR}} / L_{\odot}\right)>10.95, M_{V}<-19\right.$, $\left.\mathrm{dr}<R_{200}\right)$ vs. cluster redshift considering only blue galaxies (top) and red galaxies (bottom). The small circles show values for individual clusters, where error bars are omitted for clarity. The large circles show binned fractions for clusters. The gray triangles show the values for the GEMS field galaxies.

clusters, meaning the number of red LIRGs is normalized by a larger number of red galaxies. However, this does not fully account for the difference, and we will further investigate the differences in the red cluster/field populations in a future paper.

Spitzer studies of local clusters reveal very few galaxies with $\log _{10}\left(L_{\mathrm{IR}} / L_{\odot}\right)>10.95$ (Bai et al. 2006, 2009). In fact, the fraction of such galaxies with $M_{V}<-20.1$ within $R_{200}$ is $0 /$ 274 for Coma and 1/288 for Abell 3266 (L. Bai 2009, private communication). While Coma and Abell 3266 are more massive than the EDisCS clusters and thus might not be a fair baseline because of this mass mismatch, we find no dependence of the
LIRG fraction on cluster velocity dispersion within the EDisCS sample, and so we proceed with the comparison. In Figure 9, we show the fraction of LIRGs versus look-back time for the EDisCS and local clusters. The dashed line shows an exponential with an $e$-folding time of $2.2 \mathrm{Gyr}$. While this is by no means a unique fit to the data, it does show that the drop in LIRG fraction is consistent with an exponential decline. In their study of eight massive $0.02<z<0.83$ clusters, Saintonge et al. (2008) also find that the fraction of IR galaxies climbs steadily with redshift.

For comparison, we also show the fraction of IR galaxies in the GEMS sample in Figure 9. As an illustration, we show two curves with the field data that depict two different evolutionary scenarios. The first curve (dot-dashed line) is the same function as for the clusters, but the decline in the field is delayed by 0.9 Gyr with respect to the clusters. The second curve (dashed line) shows the field LIRG population declining at a faster rate than the cluster LIRGs; this model produces the same behavior over the epoch probed by the GEMS galaxies but predicts fewer LIRGs in local field environments. A low-redshift baseline is needed to constrain the decline rate of field LIRGs.

Comparison with previous EDisCS studies suggests that LIRGs decline at a faster rate than normal star-forming galaxies. In a study comparing three $z>0.6$ EDisCS clusters to a large sample of SDSS clusters, Finn et al. (2008) find that the fraction of $\mathrm{H} \alpha$-emitting galaxies declines by a factor of $6 \pm 3$. Comparison with results from low-redshift clusters (Bai et al. 2007, 2009) suggest that the fraction of LIRGs has decreased by a factor of $\sim 100$ during the same time period. This disparity in evolution rates is likely due to the systematic decline in star formation activity of all star-forming galaxies. For example, if we assume that the IR luminosity of all galaxies is fading at the same rate, then the IR luminosity function will shift systematically to lower luminosities with decreasing redshift (e.g., Le Floc'h et al. 2005). The most actively star-forming 


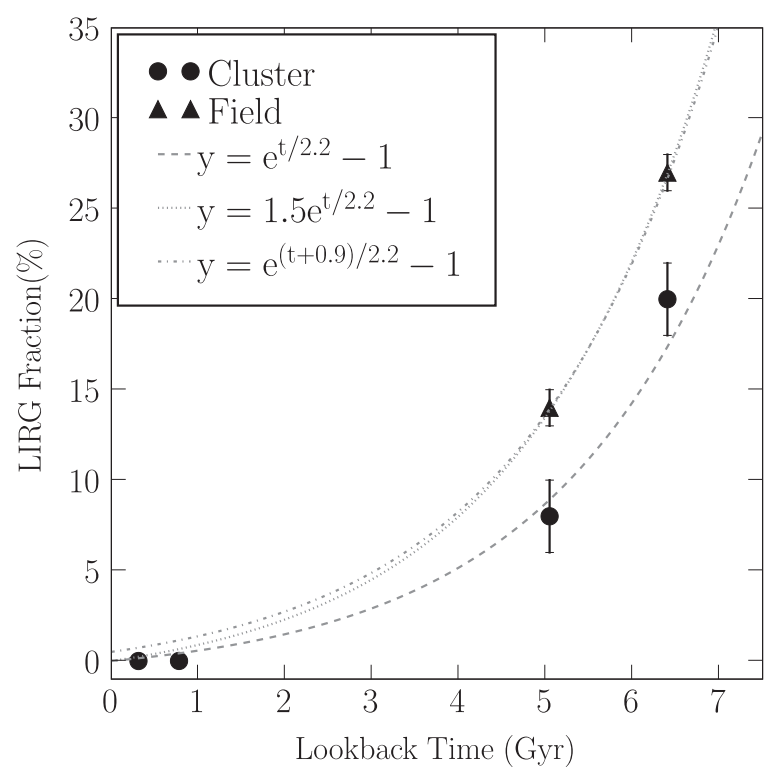

Figure 9. Fraction of IR galaxies $\left(M_{V}<-20.1, \log _{10}\left(L_{\mathrm{IR}} / L_{\odot}\right)>10.95 \mathrm{dr}<\right.$ $\left.R_{200}\right)$ vs. look-back time for clusters (circles) and field (triangles) galaxies. The dashed line shows an exponential with an $e$-folding time of $2.2 \mathrm{Gyr}$, which fits the cluster data (filled circles) reasonably well. The dot-dashed line shows the same exponential decline but delayed by $0.9 \mathrm{Gyr}$, while the dotted line shows an exponential with a faster decline rate. A low-redshift baseline is needed to better constrain the evolution of IR galaxies in the field.

galaxies - the ones we detect in the EDisCS clusters with Spitzer-will not be present at lower redshift. In contrast, the number density of galaxies at lower IR luminosities does not change as dramatically. We will explore the evolution of the cluster IR luminosity function in more detail in a future paper.

\section{DISCUSSION}

\subsection{The Connection between LIRGs and e(a) Galaxies}

The MORPHS collaboration first termed the spectral class e(a) to denote galaxies that show an A-star spectrum with emission lines (Dressler et al. 1999; Poggianti et al. 1999). These galaxies are often associated with dusty starburst galaxies in the local universe (Poggianti \& Wu 2000). Poggianti et al. (2009) use the EDisCS sample to further investigate the environments of these dusty starburst galaxies, and we compare the properties of this optically defined sample to our IR-selected galaxies. Poggianti et al. find that e(a) galaxies make up $12 \%$ of the $M_{V}<-20.1$ cluster population; we find that LIRGs make up $\sim 15 \%$ of $M_{V}<-20.1$ cluster members, entirely consistent with the assumption that e(a) galaxies are dusty starburst and are the same galaxies that we are detecting as LIRGs. Furthermore, of the LIRGs that have existing optical spectroscopy, roughly three-quarters exhibit e(a) spectra. Finally, e(a) galaxies are found least frequently in the centers of clusters. Again, this is entirely consistent with the environmental dependence we find for LIRGs. Dressler et al. (2009a) also find that Spitzer-detected galaxies in intermediate-redshift clusters exhibit predominantly e(a) spectra.

Dressler et al. (2009b) show that e(a) galaxies in a $z=0.4$ cluster have IR SFRs that are on average four times greater than SFRs derived from [O II] emission. For our sample, the median ratio of SFRs-derived $24 \mu \mathrm{m}$ emission with dust-corrected SFRs derived from [O II] is 2.9 (Vulcani et al. 2010). The large ratio of IR to optical SFRs is not surprising given the high SFRs of the IR galaxies (e.g., Zheng et al. 2006).

\subsection{Do Clusters Cause LIRGs?}

Some galaxy evolution models predict a cluster-induced burst of star formation as a galaxy falls into a cluster for the first time and the interstellar medium is compressed by the intracluster medium (e.g., Bekki \& Couch 2003). We find no evidence that clusters contain an overabundance of LIRGs relative to the field, at least within $R_{200}$. In contrast, results for other $z \sim 0.8$ galaxy clusters presented by Marcillac et al. (2007) and Bai et al. (2007) conclude that LIRGs occur more frequently in clusters. However, these authors use the surface density of LIRGs to quantify density without normalizing by the total number of cluster galaxies. Thus, their inferred overdensity of LIRGs relative to the field is likely due to the fact that the surface density of all galaxies is higher in the vicinity of the cluster. In addition, as shown in Figure 7, cluster-to-cluster variations are large, and thus large samples of clusters are needed to accurately quantify the star formation properties of clusters.

\subsection{Mechanisms to Explain Declining SFRs}

The SFRs of both field and cluster galaxies have declined dramatically since $z \sim 1$, and we have yet to address the possible mechanisms that can shut off star formation in these galaxies. This brings us full-circle to the goals outlined in Section 1.

Before proceeding we note that different mechanisms may be responsible for the star formation and morphological transformations in clusters. We know that between the epoch of the EDisCS clusters and the present universe, the fraction of cluster spirals declines while the population of S0s increases. Desai et al. (2007) find no significant change in S0 fraction within EDisCS sample, but we observe a decline in SFRs. This implies that SFRs are changing faster than morphologies, and in fact the SF and morphology evolution might be driven by two different mechanisms. This supports results of other cluster studies (Poggianti et al. 1999, 2006; Sánchez-Blázquez et al. 2009; Geach et al. 2009). In the remainder of this subsection, we focus solely on the processes that may be affecting SFRs.

From our observations, we are able to place constraints on the mechanism(s) responsible for the decline of luminous IR galaxies. We see a decline in the fraction of LIRGs in both the cluster and field samples, and the rate of the decline is the same for both samples. Furthermore, the $L_{\mathrm{IR}}$ distribution is the same for the field and cluster galaxies, but the fraction of LIRGs is lower in the clusters, similar to results for local clusters (e.g., Balogh et al. 2004; Tanaka et al. 2004; Rines et al. 2005; Finn et al. 2008). Finally, the stellar masses of the cluster LIRGs are on average higher than their field counterparts. One possible mechanism that can account for the similar evolution of field and cluster galaxies is gas depletion, where galaxies are not able to replenish their disk gas and have slowly declining star formation histories from using their available gas. The cluster environment would then have a lower fraction of star-forming galaxies because the cluster galaxies are further evolved. Figure 9 shows one plausible scenario where the decline in field LIRGs mirrors the decline in cluster LIRGs, but the field evolution is delayed by $\sim 1$ Gyr.

This scenario can also explain the higher stellar masses seen in the cluster LIRGs. For example, Figure 5 shows that SFR and stellar mass are correlated in the sense that more massive galaxies have higher SFRs. As gas depletion progresses, the SFRs of all galaxies decrease, and LIRG-levels of star formation would remain only in the most massive galaxies. In our scenario, gas depletion is more advanced in the cluster environment, and 
thus one would also expect the host galaxies of LIRGs to be more massive.

There is evidence that clusters affect the SFRs of some infalling galaxies. For example, Poggianti et al. (2009) and Poggianti et al. (1999) find an excess of post-starburst galaxies in the EDisCS clusters relative to the coeval field. These galaxies have had their star formation quenched abruptly within $1 \mathrm{Gyr}$ prior to observation, and their excess relative to the field indicates that cluster-specific processes are altering the star formation properties of some infalling galaxies. The excess of post-starburst galaxies in clusters is small, and we do not have a large enough sample to detect a small differential evolution between the cluster and field LIRG fraction.

Rudnick et al. (2009) provide further evidence that clusters are actively altering infalling galaxies; they find that the red sequence appears to build up more rapidly in clusters than in the field. In addition, De Lucia et al. (2007) find that the rate at which the red sequence builds up depends weakly on cluster velocity dispersion, although the interpretation of the observational findings depends on assumptions regarding the nature of the underlying mass/luminosity function of galaxies in various environments.

\section{CONCLUSIONS}

We present Spitzer MIPS observations of sixteen $0.4<z<$ 0.8 EDisCS clusters. This is the first large $24 \mu \mathrm{m}$ survey of clusters at intermediate redshift and represents a significant increase in the census of SFRs in dense environments. The limits of the $24 \mu \mathrm{m}$ imaging are such that we are sensitive to only the brightest IR galaxies, and so our sample contains mainly LIRGs rather than normal star-forming galaxies. Our major results are summarized below.

1. We calculate $L_{\mathrm{IR}}$ for the clusters members and compare to a large sample of coeval field galaxies from the literature. While the clusters contain a lower fraction of IR-emitting galaxies, the distribution of $L_{\mathrm{IR}}$ for the cluster galaxies is identical to that of the field galaxies.

2. The stellar masses of the EDisCS LIRGs are systematically higher than the stellar masses of the GEMS galaxies.

3. Approximately, $\sim 80 \%$ of the IR galaxies live in the blue cloud and the remaining $20 \%$ lie on the red sequence.

4. The majority of LIRGs have optical spectra that are dominated by A-stars and show some signs of modest ongoing star formation as determined by [O II] emission (i.e., e(a); Poggianti et al. 1999, 2008). SFRs derived from IR emission are much greater than those inferred from optical emission, with a median SFR(IR)/SFR(OII) of 2.9 (Vulcani et al. 2010).

5. LIRGs avoid the centers of clusters; the fraction of IR galaxies is lowest near the cluster center $\left(\mathrm{dr}<0.5 \times R_{200}\right)$ and remains below the field value at least out to $1.5 \times R_{200}$.

6 . The fraction of IR galaxies decreases significantly over the $2.4 \mathrm{Gyr}$ interval spanned by our sample, and the rate of the decline is the same for the cluster and field populations. Comparison with IR studies of local clusters shows that the evolution of the cluster LIRGs is consistent with an exponential decline with an $e$-folding time of $2.2 \mathrm{Gyr}$.

7. SFRs are declining faster than morphologies are transforming, consistent with numerous previous studies.

8. The similar decline of field and cluster LIRGs suggests that the mechanism driving the global decline of SFRs is the same in the cluster and field environments. We find gas depletion to be the most likely candidate, where the decline in the field is delayed by $\sim 1$ Gyr with respect to the clusters.

R.A.F. thanks L. Bai for useful discussions and for aiding with the comparison to her work. B.M.P. acknowledges financial support from ASI contract I/016/07/0. We thank the anonymous referee for suggestions that significantly improved the paper. This work is based on observations made with the Spitzer Space Telescope, which is operated by the Jet Propulsion Laboratory, California Institute of Technology under a contract with NASA. Support for this work was provided by NASA through an award issued by JPL/Caltech. The Dark Cosmology Centre is funded by the Danish National Research Foundation.

\section{REFERENCES}

Bai, L., Rieke, G. H., Rieke, M. J., Christlein, D., \& Zabludoff, A. I. 2009, ApJ, 693, 1840

Bai, L., Rieke, G. H., Rieke, M. J., Hinz, J. L., Kelly, D. M., \& Blaylock, M. 2006, ApJ, 639, 827

Bai, L., et al. 2007, ApJ, 664, 181

Balogh, M., et al. 2004, MNRAS, 348, 1355

Balogh, M. L., \& Morris, S. L. 2000, MNRAS, 318, 703

Balogh, M. L., Morris, S. L., Yee, H. K. C., Carlberg, R. G., \& Ellingson, E. 1997, ApJ, 488, L75

Balogh, M. L., Morris, S. L., Yee, H. K. C., Carlberg, R. G., \& Ellingson, E. 1999, ApJ, 527, 54

Balogh, M. L., Schade, D., Morris, S. L., Yee, H. K. C., Carlberg, R. G., \& Ellingson, E. 1998, ApJ, 504, L75

Bekki, K., \& Couch, W. J. 2003, ApJ, 596, L13

Bell, E. F., McIntosh, D. H., Katz, N., \& Weinberg, M. D. 2003, ApJS, 149, 289

Bell, E. F., et al. 2005, ApJ, 625, 23

Bertin, E., \& Arnouts, S. 1996, A\&AS, 117, 393

Blanton, M. R., \& Moustakas, J. 2009, ARA\&A, 47, 159

Bridge, C. R., et al. 2007, ApJ, 659, 931

Cassata, P., et al. 2007, ApJS, 172, 270

Clowe, D., et al. 2006, A\&A, 451, 395

Connolly, A. J., Szalay, A. S., Dickinson, M., Subbarao, M. U., \& Brunner, R. J. 1997, ApJ, 486, L11

Cooper, M. C., et al. 2007, MNRAS, 376, 1445

Cooper, M. C., et al. 2008, MNRAS, 383, 1058

Cucciati, O., et al. 2006, A\&A, 458, 39

Dale, D. A., \& Helou, G. 2002, ApJ, 576, 159

De Lucia, G., Springel, V., White, S. D. M., Croton, D., \& Kauffmann, G. 2006, MNRAS, 366, 499

De Lucia, G., et al. 2007, MNRAS, 374, 809

Desai, V., et al. 2007, ApJ, 660, 1151

Donley, J. L., Rieke, G. H., Pérez-González, P. G., Rigby, J. R., \& AlonsoHerrero, A. 2007, ApJ, 660, 167

Dressler, A., Oemler, A., Gladders, M. G., Bai, L., Rigby, J. R., \& Poggianti, B. M. 2009a, ApJ, 699, L130

Dressler, A., Rigby, J., Oemler, A., Fritz, J., Poggianti, B. M., Rieke, G., \& Bai, L. 2009b, ApJ, 693, 140

Dressler, A., Smail, I., Poggianti, B. M., Butcher, H., Couch, W. J., Ellis, R. S., \& Oemler, A. J. 1999, ApJS, 122, 51

Elbaz, D., et al. 2007, A\&A, 468, 33

Ellingson, E., Lin, H., Yee, H. K. C., \& Carlberg, R. G. 2001, ApJ, 547, 609

Fazio, G. G., et al. 2004, ApJS, 154, 10

Finn, R. A., Balogh, M. L., Zaritsky, D., Miller, C. J., \& Nichol, R. C. 2008, ApJ, 679,279

Finn, R. A., Zaritsky, D., \& McCarthy, D. W. 2004, ApJ, 604, 141

Finn, R. A., et al. 2005, ApJ, 630, 206

Flores, H., et al. 1999, ApJ, 517, 148

Gallego, J., Zamorano, J., Aragon-Salamanca, A., \& Rego, M. 1995, ApJ, 455, L1

Geach, J. E., et al. 2006, ApJ, 649, 661

Geach, J. E., Smail, I., Moran, S. M., Treu, T., \& Ellis, R. S. 2009, ApJ, 691, 783

Gerke, B. F., et al. 2007, MNRAS, 376, 1425

Giavalisco, M., et al. 2004, ApJ, 600, L103

Gómez, P. L., et al. 2003, ApJ, 584, 210

Gordon, K. D., et al. 2005, PASP, 117, 503

Halliday, C., et al. 2004, A\&A, 427, 397

Hashimoto, Y., Oemler, A. J., Lin, H., \& Tucker, D. L. 1998, ApJ, 499, 589 
Hopkins, A. M. 2004, ApJ, 615, 209

Houck, J. R., et al. 2004, ApJS, 154, 18

Kauffmann, G., White, S. D. M., Heckman, T. M., Ménard, B., Brinchmann, J., Charlot, S., Tremonti, C., \& Brinkmann, J. 2004, MNRAS, 353, 713

Kennicutt, R. C. 1998, ARA\&A, 36, 189

Kessler, M. F., et al. 1996, A\&A, 315, L27

Kodama, T., Balogh, M. L., Smail, I., Bower, R. G., \& Nakata, F. 2004, MNRAS, 354,1103

Lacey, C. G., Baugh, C. M., Frenk, C. S., Silva, L., Granato, G. L., \& Bressan, A. 2008, MNRAS, 385, 1155

Larson, R. B., Tinsley, B. M., \& Caldwell, C. N. 1980, ApJ, 237, 692

Le Fèvre, O., et al. 2000, MNRAS, 311, 565

Le Floc'h, E., et al. 2005, ApJ, 632, 169

Lewis, I., et al. 2002, MNRAS, 334, 673

Lilly, S. J., Le Fevre, O., Hammer, F., \& Crampton, D. 1996, ApJ, 460, L1

Lotz, J. M., et al. 2008, ApJ, 672, 177

Madau, P., Ferguson, H. C., Dickinson, M. E., Giavalisco, M., Steidel, C. C., \& Fruchter, A. 1996, MNRAS, 283, 1388

Marcillac, D., Rigby, J. R., Rieke, G. H., \& Kelly, D. M. 2007, ApJ, 654, 825

Metcalfe, L., Fadda, D., \& Biviano, A. 2005, Space Sci. Rev., 119, 425

Milvang-Jensen, B., et al. 2008, A\&A, 482, 419

Noeske, K. G., et al. 2007, ApJ, 660, L43

Papovich, C., et al. 2004, ApJS, 154, 70

Poggianti, B. M., \& Wu, H. 2000, ApJ, 529, 157

Poggianti, B. M., Smail, I., Dressler, A., Couch, W. J., Barger, A. J., Butcher, H., Ellis, R. S., \& Oemler, A. J. 1999, ApJ, 518, 576

Pello, R., et al. 2009, A\&A, 508, 1173

Poggianti, B. M., et al. 2008, ApJ, 684, 888

Poggianti, B. M., et al. 2009, ApJ, 693, 112
Poggianti, B. M., et al. 2006, ApJ, 642, 188

Rieke, G. H., et al. 2004, ApJS, 154, 25

Rines, K., Geller, M. J., Kurtz, M. J., \& Diaferio, A. 2005, AJ, 130, 1482

Rix, H.-W., et al. 2004, ApJS, 152, 163

Robaina, A. R., et al. 2009, ApJ, 704, 324

Rudnick, G., et al. 2009, ApJ, 700, 1559

Saintonge, A., Tran, K., \& Holden, B. P. 2008, ApJ, 685, L113

Sánchez-Blázquez, P., et al. 2009, A\&A, 499, 47

Sanders, D. B., \& Mirabel, I. F. 1996, ARA\&A, 34, 749

Serjeant, S., et al. 2008, MNRAS, 386, 1907

Steidel, C. C., Adelberger, K. L., Giavalisco, M., Dickinson, M., \& Pettini, M. 1999, ApJ, 519, 1

Stern, D., et al. 2005, ApJ, 631, 163

Tanaka, M., Goto, T., Okamura, S., Shimasaku, K., \& Brinkmann, J. 2004, AJ, 128,2677

Tran, K., Saintonge, A., Moustakas, J., Bai, L., Gonzalez, A. H., Holden, B. P., Zaritsky, D., \& Kautsch, S. J. 2009, ApJ, 705, 809

Treyer, M. A., Ellis, R. S., Milliard, B., Donas, J., \& Bridges, T. J. 1998, MNRAS, 300, 303

Vulcani, B., Poggianti, B. M., Finn, R. A., Rudnick, G., Desai, V., \& Bamford, S. 2010, ApJ, 710, L1

Werner, M. W., et al. 2004, ApJS, 154, 1

White, S. D. M., et al. 2005, A\&A, 444, 365

Wilson, G., Cowie, L. L., Barger, A. J., \& Burke, D. J. 2002, AJ, 124, 1258

Wolf, C., Dye, S., Kleinheinrich, M., Meisenheimer, K., Rix, H.-W., \& Wisotzki, L. 2001, A\&A, 377, 442

Wolf, C., et al. 2004, A\&A, 421, 913

Zheng, X. Z., Bell, E. F., Rix, H.-W., Papovich, C., Le Floc'h, E., Rieke, G. H., \& Pérez-González, P. G. 2006, ApJ, 640, 784 